\title{
Proton beam therapy for malignant pleural mesothelioma
}

\author{
Shahed N. Badiyan, Jason K. Molitoris, Mingyao Zhu, Erica Glass, Tejan Diwanji, Charles B. Simone II \\ University of Maryland School of Medicine, Baltimore, MD, USA \\ Contributions: (I) Conception and design: SN Badiyan, CB Simone 2nd; (II) Administrative support: None; (III) Provision of study materials or \\ patients: All authors; (IV) Collection and assembly of data: All authors; (V) Data analysis and interpretation: SN Badiyan, CB Simone 2nd; (VI) \\ Manuscript writing: All authors; (VII) Final approval of manuscript: All authors. \\ Correspondence to: Shahed N. Badiyan, MD. Assistant Professor, Radiation Oncology, 850 W. Baltimore St, Baltimore, MD 21210, USA. Email: \\ shahedbadiyan@gmail.com.
}

\begin{abstract}
Malignant pleural mesothelioma (MPM) is a rare disease with a poor prognosis. Surgical techniques have made incremental improvements over the last few decades while new systemic therapies, including immunotherapies, show promise as potentially effective novel therapies. Radiation therapy has historically been used only in the palliative setting or as adjuvant therapy after extrapleural pneumonectomy, but recent advances in treatment planning and delivery techniques utilizing intensity-modulated radiation therapy and more recently pencil-beam scanning (PBS) proton therapy, have enabled the delivery of radiation therapy as neoadjuvant or adjuvant therapy after an extended pleurectomy and decortication or as definitive therapy for patients with recurrent or unresectable disease. In particular, PBS proton therapy has the potential to deliver high doses of irradiation to the entire effected pleura while significantly reducing doses to nearby organs at risk. This article describes the evolution of radiation therapy for MPM and details how whole-pleural PBS proton therapy is delivered to patients at the Maryland Proton Treatment Center.
\end{abstract}

Keywords: Malignant pleural mesothelioma (MPM); radiation; proton beam therapy; pencil-beam scanning (PBS); spot scanning

Submitted Dec 25, 2017. Accepted for publication Mar 28, 2018.

doi: 10.21037/tlcr.2018.04.07

View this article at: http://dx.doi.org/10.21037/tlcr.2018.04.07

\section{Introduction}

Malignant pleural mesothelioma (MPM) is a rare disease with poor overall survival (OS) and limited effective treatment options. Much progress has been made over the past decade with the development of novel treatments, including new chemotherapeutic and immunotherapeutic regimens as well as new surgical techniques. Despite this progress, survival has remained poor, and the median OS remains approximately 1 year (1).

The incidence rate of MPM is 2,500-3,000 cases per year in the United States (1). The incidence is higher in men and in patients over 65 years (2). Asbestos exposure is the most significant risk factor for the development of MPM, and previous exposure, including occupational, can be traced in most patients (3). Germ-line mutation of the BRCA1associated protein 1 (BAP1) tumor suppressor gene and (4) prior exposure to ionizing radiation are also associated with increased risk of developing MPM $(5,6)$.

Most patients with MPM are diagnosed at advanced stages (7). In cases where thoracentesis is performed, cytology is often non-confirmatory (8). The optimal procedure for more definitive diagnosis and more adequate histologic subtyping is thoracoscopy with pleural biopsy (9). Determining the histologic subtype is of utmost importance to guide therapeutic options and to inform prognosis. The three major histologic subtypes of MPM are epithelioid, sarcomatoid, and biphasic, in which both epithelioid and sarcomatoid components are present. Epithelioid histology is the most common and is associated with a better prognosis and higher responses to treatment when compared to sarcomatoid and biphasic histologies (10). In the Surveillance Epidemiology and End Results Program (SEER) database, 3-year survival for epithelioid was $15.9 \%$, 
biphasic $9.3 \%$, and sarcomatoid 0\% (11).

Another key aspect of diagnosis is accurate staging, including nodal staging and assessment for distant metastases. TNM staging is important for predicting OS. Increasing $\mathrm{T}$ stage is associated with worse survival (12), and the presence of nodal disease is independently associated with poor outcomes regardless of histologic subtype. Median survival for patients with N1 or higher disease is approximately half that of patients with N0 disease (13). Distant metastases are seen infrequently at presentation (14) but can occur in virtually any tissue site including unusual sites such as the gingiva, skin, and brain (15-17).

MPM often has a high symptom burden, and many patients present with fatigue, dyspnea and pleural effusions that may be initially attributed to other causes, delaying diagnosis of the underlying malignancy. These symptoms can be debilitating and difficult to manage. Pleuritic pain can be intractable and often responds poorly to opioid therapy (18). Dyspnea can result from recurrent pleural effusions, and thoracentesis may ameliorate symptoms. Talc pleurodesis can also be effective, although it may be less effective for MPM compared to effusions caused by lung cancers (19). Palliative pleurectomies can also be considered.

Disease-directed treatment options for more definitive management of MPM include systemic therapy, surgery, and radiation therapy. Surgery is an important treatment modality that may, in select cases, most optimally allow for prolonged survival, but only a minority of patients are medically operable and technically resectable $(20,21)$. Two definitive surgical procedures are considered standard approaches for MPM. The first, extrapleural pneumonectomy (EPP), involves removal of the entire ipsilateral lung, affected pleura and portions of the diaphragm and pericardium. The second, extended pleurectomy and decortication (PD), involves removal of the affected pleura and generally also the diaphragm and pericardium but retains the ipsilateral lung (22). Disease recurrence after either definitive surgical procedure alone is very common; as such, intraoperative adjuvant therapies (23), as well as additional treatments including chemotherapy and/or radiation therapy are generally recommended $(24,25)$. In patients with unresectable disease, systemic chemotherapy can improve survival and symptom burden. The doublet regimen of cisplatin and pemetrexed improves OS and time to progression as compared to cisplatin alone (26). The addition of bevacizumab to the platinum/pemetrexed doublet has also been shown to increase OS (27). More recently, the KEYNOTE-028 trial demonstrated activity of pembrolizumab, a programmed death-1 (PD-1) inhibitor, in patients with programmed death ligand-1 (PD-L1) positive MPM (28). Further research is ongoing to elucidate the role of immunotherapy in the management of mesothelioma.

The role of radiation therapy has evolved over the last few decades from a treatment modality used solely for palliative purposes to one used as adjuvant therapy after EPP and more recently as neoadjuvant or adjuvant therapy in those who undergo PD. This review explores the evolution of radiation therapy focusing on the technological advancements in the field that have enabled more sophisticated and aggressive radiation therapy treatments culminating in the recent advent of pencil-beam scanning (PBS) proton therapy that may allow for the safe delivery of definitive radiation therapy to patients who are not candidates for EPP or PD.

\section{Radiation therapy indications for mesothelioma}

Radiation delivery in the treatment of MPM is technically challenging, which has limited its use in multi-modality management of MPM. Several lines of evidence, however, suggest that radiation could be beneficial in the treatment of MPM. First, in vitro mesothelioma cells have similar to improved radiosensitivity compared with non-small cell lung cancer $(29,30)$. Also, within palliative treatments, there does appear to be a dose response relationship in clinical experiences (31). Finally, radiation to areas at high risk for local failure, including the thoracic cavity surgical tract and access sites, may significantly reduce local failure rates.

As most patients present with advanced disease, radiation therapy is most commonly used in the palliative setting for MPM. Radiation therapy provides effective pain control and in one series of 29 treatments to 17 patients, 4 of 6 patients treated to doses above 40 Gy experienced significant symptom relief, while only one patient treated to a lower dose had improvement (31). In another study, improved local response was observed when treating with 36 Gy in 9 fractions compared to 30 Gy in 8 or more fractions $(50 \%$ vs. 39\%) (32). These data argue that radiation therapy can be effective for pain control and higher doses may lead to improved outcomes.

Another common use of radiation therapy in the treatment of MPM is prophylactic treatment to biopsy and drain sites. Several randomized trials have been performed with mixed outcomes. A French study randomized 40 
patients to radiation (21 Gy in 3 fractions) or no radiation to all instrumentation sites. They reported no local failures in the patients who received radiation compared to failures in $40 \%(n=8)$ of patients in the non-irradiated group (33). However, a randomized trial of 61 patients performed in England reported no improvement in track site seeding with radiation to $21 \mathrm{~Gy}$ in 3 fractions (34). Another study performed in Australia used a single 10-Gy fraction delivered with $9 \mathrm{MeV}$ electrons and found no difference in recurrences, although dose fractionation and penetration could be confounding factors (35). Despite the lack of consensus from these studies, many offer radiation because track site recurrences can be morbid and treatment is simple with minimal side-effects.

A single arm phase II study of hypofractionated neoadjuvant radiation therapy prior to EPP was conducted by investigators at Princess Margaret Hospital. Patients with MPM who were candidates for EPP received hypofractionated irradiation to the entire effected lung and pleura to 25 Gy in 5 fractions with a simultaneous integrated boost to $30 \mathrm{~Gy}$ to gross disease. Patients then went on to undergo an EPP within 7 days of completing radiation. Patients with positive lymph nodes also received adjuvant chemotherapy. They reported an impressive median OS of 36 months (36).

With the exception of a limited number of neoadjuvant radiation studies, the use of radiation in the non-palliative setting has largely been delivered in the adjuvant setting and has evolved over the past 10-15 years with advances in surgical approaches and increasingly conformal radiation technologies.

\section{Adjuvant photon radiation therapy}

\section{Photon therapy after EPP}

Traditionally, adjuvant radiation therapy for MPM was delivered with a mix of photons and electrons using matched fields (37). However, over the past two decades, intensitymodulated radiation therapy (IMRT) has largely supplanted conventional techniques. The putative dosimetric benefits of IMRT after EPP were evaluated by Krayenbuehl et al. who demonstrated in a small comparative planning study that IMRT improved target volume coverage at the expense of increased doses to the surrounding organs (38). This improvement in target coverage is particularly important given early data suggested poor local control with threedimensional (3D) techniques (37). Modern IMRT series have demonstrated local failure rates between $8-46 \%$ (39-45). A primary concern with treating these patients, however, is the potentially high rates of toxicities.

In particular, IMRT can increase dose to the remaining contralateral lung over conventional 3D techniques (38). Clinically, the combined experience of Dana-Farber Cancer Institute and Brigham and Women's Hospital suggested that this increased lung dose may in fact contribute to higher than expected rates of fatal pneumonitis (46\%) (46). In their combined retrospective review of 13 patients, Allen et al. also identified multiple dosimetric parameters including V5, V20, and mean lung dose (MLD) that were associated with increased risk of fatal pneumonitis and consequently should be considering when using IMRT (46). Subsequent published retrospective experiences from Copenhagen University Hospital, Duke University, and MD Anderson Cancer Center have supported the significance of one or more of these dosimetric parameters $(39,47,48)$. The Danish study additionally found a potential correlation with the lung V10 metric (47). These established predictors of pulmonary toxicities raised concerns about the use of IMRT in the setting and has led to work in defining guidelines for adequate thresholds. However, treatment planning is not an exact science, and in an effort to demonstrate this, the group from Duke University retrospectively analyzed their cohort of 30 patients. They subdivided this set into two groups of 15 patients and evaluated various planning parameters including treatment volumes and dose-volume histogram (DVH) metrics. Interestingly, even in the era where the risk of complications was known, they demonstrated that MLD and V5 decreased with time, suggesting that increased experience may allow for improved optimization of planning and dosimetry (40). Other modulation techniques, including arc therapy and helical tomotherapy, may have the potential to improve dose homogeneity and coverage but tend to increase the low dose bath (i.e., V5) to the contralateral lung. Regardless, early clinical experience has demonstrated such an approach can still be relatively safe (41).

Lastly, it is important to note that patients undergoing this treatment tend to have other acute treatment-induced toxicities, such as nausea/emesis and esophagitis as well. These rates vary widely amongst the retrospective series, but a recent randomized trial revealed rates of $11 \%$ and $7 \%$, respectively (49). Dosimetric parameters have been associated with the development of these toxicities, but due to the target volume, these can be difficult to attain while maintaining adequate coverage regardless of which photon therapy technique is used. 


\section{Photon therapy after PD}

Unlike radiation therapy after EPP, adjuvant radiation in the setting of $\mathrm{PD}$ is complicated by the presence of the ipsilateral lung. As such, conventional matched photonelectron plans must be modified to block the central portion of the lungs, and additional patched electron fields are used to irradiate those corresponding anterior and posterior central regions of the pleura $(50,51)$. Clinical results from patients treated over three decades [1974-2003] at Memorial Sloan-Kettering Cancer Center, however, revealed local control at 1 year of a meager $42 \%$ (51). This was also despite the fact that almost half of the patients also received additional dose via brachytherapy (matched peripheral dose of 160 Gy) (51). Furthermore, the technique was also found to have significant acute toxicities (51).

Due to the unfavorable results with conventional radiation both in terms of local control and toxicity, some researchers have attempted to develop IMRT techniques in the setting of PD. Initial work from Rosenzweig et al. revealed that such a method of treatment could be feasible and safe (52). They utilized eight equally spaced beams over an angle of 220-240 degrees to prevent irradiation beam entrance through the contralateral lung. Furthermore, they found relatively low rates of acute toxicities with only $20 \%$ grade $\geq 3$ pneumonitis (52). Unfortunately, updated results revealed that local control rates remained low with 2-year actuarial local control of $40 \%$ in patients who received PD (53). This rate was further diminished to $17 \%$ in patients receiving subtotal surgery or biopsy alone (53). These results eventually led to the phase II IMPRINT (Hemithoracic IntensityModulated Pleural Radiation Therapy) trial, which once again confirmed that PD with adjuvant IMRT still resulted in sub-optimal local control (59\% of patients had treatment failure within the radiation field), but only $2 / 27$ patients developed grade 3 radiation pneumonitis and there were no instances of grade 4+ pneumonitis (54).

Alternative techniques of intensity modulation, such as tomotherapy, have also been attempted in this setting with slightly more favorable results (55-57). A prospective study of 28 patients undertaken in Italy revealed that tomotherapy could be used safely with potentially lower side effects, with only $7 \%$ developing grade $\geq 3$ pneumonitis, and no grade 5 toxicities were identified. Local control and survival results are pending (55). Additionally, a retrospective study of patients at UCLA suggested that local control may be better with tomotherapy than three-dimensional conformal radiotherapy (3DCRT) (56).

\section{Proton beam therapy}

The dose-deposition properties of proton therapy make the treatment particularly well-suited for the treatment of MPM. Unlike a photon beam that deposits energy through the entire length of the beam and deposits the majority of its dose just underneath the skin, a proton beam has a lower entrance dose and deposits the majority of its dose at the end of its range; a phenomenon called the Bragg peak. This dose deposition profile results in lack of exit dose and superior sparing of nearby normal tissues compared to photon therapy (58). Early proton therapy machines required the use of custommade scattering devices, collimators, and apertures to scatter and shape the beam to the shape of the target (i.e., passive scattering proton therapy). However, a newer technique, PBS, eliminates the need for custom-made devices by using steering magnets to paint proton beam spots layer-by-layer over the target volume. This technology not only improves target coverage of complex shapes compared to passive scattering proton therapy but also enables the delivery of intensitymodulated proton therapy (IMPT) (58), although care must be taken with the delivery of PBS since it can be more susceptible to intrafractional tumor motion and interplay effects, as well as interfractional tumor and normal tissue changes compared with passive scattering proton therapy (59). This underscores the importance of volumetric imaging for patient alignment before treatment and to assess for anatomical changes during treatment when delivering PBS (60).

Mesothelioma target volumes for radiation therapy, especially whole pleura target volumes, are usually large and complexly shaped. Organs abutting the target volumes such as the lungs, heart, liver, kidneys and spinal cord can result in limitations in the ability to deliver definitive doses of radiation therapy; however, PBS proton therapy can in many cases overcome these challenges. A number of dosimetric studies and, more recently, clinical data on proton beam therapy for MPM have demonstrated superior normal tissue sparing compared to photon techniques. The remainder of this review will examine the current literature on proton beam therapy for MPM and describe how wholepleural intensity-modulated proton therapy (WP-IMPT) is delivered at the University of Maryland.

\section{Dosimetric studies}

A pair of comparative planning studies have demonstrated significant reductions in doses to organs at risk (OARs) when treating mesothelioma with PBS proton therapy 
compared to IMRT. Investigators in Switzerland selected eight patients with MPM that had undergone EPP followed by IMRT and re-planned their treatments with PBS proton therapy. Coverage of the planning target volume (PTV), as measured by V95, was significantly improved in the PBS plans. In addition, mean doses to the spinal cord, contralateral (2.7 vs. $0.1 \mathrm{~Gy}, \mathrm{P}<0.01)$ and ipsilateral kidneys (11.8 vs. 7.0 Gy, $\mathrm{P}=0.02$ ), contralateral lung (4.6 vs. $0.4 \mathrm{~Gy}$, $\mathrm{P}<0.01)$, heart (25 vs. $6 \mathrm{~Gy}, \mathrm{P}<0.01)$, and liver $(13.2$ vs. 3.7 Gy, $\mathrm{P}=0.01$ ) were significantly decreased with $\mathrm{PBS}$. The mean contralateral lung V5Gy (27.5\% vs. 1.5\%), V13Gy ( $7.1 \%$ vs. $0.7 \%$ ) and V20Gy (2.9\% vs. $0.5 \%)$ were also significantly improved with PBS (all $\mathrm{P}<0.01)$ (61).

Similarly, Lorentini treated seven patients with IMRT after EPP and retrospectively re-planned their treatments with PBS proton therapy. They also found significant reductions in mean doses to the esophagus (36.9 vs. $26.2 \mathrm{~Gy}$, $\mathrm{P}<0.001)$, heart (24.6 vs. 12.1 Gy, $\mathrm{P}<0.001)$, contralateral lung (6.1 vs. $0.2 \mathrm{~Gy}, \mathrm{P}<0.001)$, ipsilateral and bilateral kidneys, and liver (24.5 vs. $14.2 \mathrm{~Gy}, \mathrm{P}<0.01)$ with $\mathrm{PBS}$ proton therapy compared to IMRT. In addition, normal tissue complication probability (NTCP) modeling was used to estimate the risk reduction in toxicities to OARs using PBS proton therapy and IMRT. Results suggest significantly reduced risks of radiation-induced liver disease, kidney injury, and acute esophagitis with the use of PBS proton therapy as compared to IMRT (62).

\section{Clinical data}

Despite this strong dosimetric rationale for proton therapy, clinical data on the use of proton therapy to treat MPM are limited. There are clinical series on proton therapy for the treatment of locally advanced non-small cell lung cancer, with the goals of improving quality of life (63) or minimizing toxicities, both in the definitive (64-66) and adjuvant (67) setting. There are also series on the proton therapy treatment of small cell lung cancer (68), thymic malignancies (69), and recurrent thoracic malignancies (70). However, reports of proton therapy for MPM to date are limited to small single-institution reports.

The first published clinical report using proton therapy to treat MPM was a case series from MD Anderson Cancer Center. Among seven patients with lung-intact MPM, 4 were treated with PBS proton therapy and 3 received IMRT. In a plan comparison, the PBS plans achieved lower mean heart, esophagus, liver, contralateral lung, and ipsilateral kidney doses than the IMRT plans. Clinically, all patients receiving proton therapy tolerated treatment well and did not require treatment breaks, although outcomes data are pending (71).

University of Pennsylvania investigators presented the largest experience to date using proton therapy to treat MPM. Those investigators evaluated 16 patients treated with 17 proton therapy courses. Patients were predominantly male $(81 \%)$ and Caucasian (100\%) with epithelial histological subtype (82\%) and stages III-IV disease (94\%). Median age was 69.8 years old at the time of proton therapy, which was delivered a median of 11.1 months after MPM diagnosis (range, 3.5-69.3 months). All patients received pemetrexed plus cisplatin or carboplatin prior to $(\mathrm{n}=15)$ or concurrent with $(\mathrm{n}=1)$ proton therapy. Proton therapy was administered as adjuvant therapy following lung-sparing radical pleurectomy $(n=8)$, to sites of gross disease following progression on systemic therapy $(\mathrm{n}=8)$, or as initial definitive therapy with concurrent chemotherapy $(\mathrm{n}=1)$. Patients were treated to a median dose of 51.75 Gy [cobalt grey equivalent (CGE)] in 2.0 Gy (CGE) daily fractions (range 50.0-75.0 Gy/1.8-2.5 Gy). All patients had durable local control throughout the follow-up period at a median follow-up of $>5$ months from proton therapy completion. At the time of reporting, the median OS for the cohort had not yet been reached, and no patient developed any acute or late grade $\geq 3$ toxicities. Across the 17 proton therapy courses, acute grade 2 toxicities included radiation dermatitis $(n=8)$, dysphagia/esophagitis $(n=4)$, anorexia $(n=3)$, fatigue $(n=2)$, and cough $(n=1)$. Late grade 2 toxicities included a single patient with radiation pneumonitis (6\%). Additionally, patients had a modest improvement in Eastern Cooperative Oncology Group (ECOG) performance score from proton therapy beginning to end (mean, 1.2 to 0.9 ) (72).

Most recently, investigators from University of Washington School of Medicine reported on a case series of three patients with MPM treated with PBS to 54-66 Gy after EPP. In matched volumetric-modulated arc therapy photon plans for these three patients, PBS proton therapy achieved considerably lower doses to OARs. Clinically, none of the three patients developed radiation pneumonitis or required a treatment break. Acute grade 2 toxicities were limited to nausea $(\mathrm{n}=1)$ and dermatitis $(\mathrm{n}=2)(73)$.

\section{Physics considerations}

\section{Simulation and motion management}

At the University of Maryland, Maryland Proton Treatment Center WP-IMPT is being delivered to patients without 


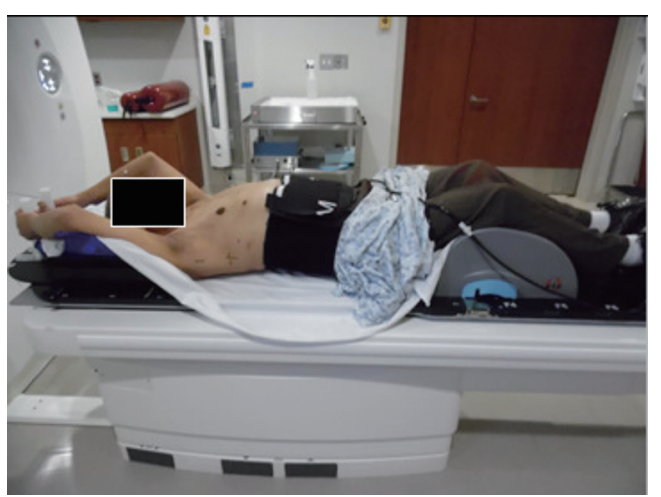

Figure 1 Malignant pleural mesothelioma patient set-up at simulation at the Maryland Proton Treatment Center demonstrating supine positioning with Vac-Lok bag, wing-board, knee wedge and abdominal compression belt.

distant metastatic disease who are not candidates for surgical intervention and have limited remaining systemic therapy options. Patients are simulated for treatment in the supine position with arms up and legs straight. Customized Vac-Lok bags are placed along with a wing-board or arm shuttle for arm immobilization, and knee wedges for leg immobilization. The wing-board, arm shuttle, and knee wedge are indexed to the computed tomography (CT) table, which has the same index position as the treatment table (Figure 1).

\section{Planning and optimization}

To account for internal patient motion, a four-dimensional (4D) CT scan with a 3-mm slice thickness is acquired from the mid cervical spine to $5 \mathrm{~cm}$ below the liver and kidneys. When necessary, a compression belt is utilized to reduce diaphragmatic motion, but it is frequently not necessary due to a fixed hemi-diaphragm in patients with extensive gross pleural disease. The target [gross tumor volume (GTV) and clinical target volume (CTV)] and the OARs are contoured on the average CT image (CT_avg), then the internal target volume (ITV) for the targets [internal GTV (iGTV) and internal CTV (iCTV)] are also contoured on the CT avg with margins added to encompass the targets on all 10 phased CT images. The treatment plans are created on the average CT image, and also evaluated on the $0 \%$ and $50 \%$ phases to ensure adequate coverage.

Two-fields are typically used [sometimes with a simultaneously integrated boost (SIB)], one from anteriorposterior (AP) direction, the other from posterior-anterior (PA) direction, and both fields may be slightly oblique from the ipsilateral side, i.e., left posterior oblique (LAO) and left anterior oblique (LPO) for left-sided target, and vice versa. Multiple-field optimization (MFO) treatment planning is used to maximize the sparing of the critical organs, e.g., the heart, the liver, kidneys, and lungs (Figure 2). Due to the shallow depth of the target, range shifters with a water equivalent thickness of $5.7 \mathrm{~cm}$ are inserted for all beams. The air gap is kept at $15 \mathrm{~cm}$ to avoid collisions.

Coverage to the PTV (approximately $5-\mathrm{mm}$ expansion from the larger ITV or iCTV) is necessary to ensure the coverage of the ITV when setup and proton range uncertainties are considered. This PTV is then partitioned into sub-volumes as the field-specific target for each field to minimize proton spots traversing through critical OARs. The total number of energy layers needed to cover the fieldtarget varies from 35 to 50, depending on target size and beam angle. To evaluate plan robustness, perturbed doses are calculated for 12 scenarios, each includes one isocenter offset ( $5 \mathrm{~mm}$ in 1 of the 6 primary anatomic directions) and one range variation (3.5\%, positive or negative). The DVH of the nominal plan (solid lines) and the perturbed doses (dotted lines) are evaluated before approving the plan (Figure 3).

For the patients treated at our center, typical achieved doses to the OARs are: heart V $30<20 \%, \mathrm{~V} 45<15 \%$, and $\mathrm{D}(0.3 \mathrm{cc})<60$ Gy; total lung $\mathrm{D}_{\text {mean }}<17 \mathrm{~Gy}, \mathrm{~V} 50=10-20 \%$; contralateral lung DMean <4 Gy, V20<5\%; liver V30 $<30 \%$ (for patients with right-sided disease); ipsilateral kidney Dmean $<12$ Gy. There is no dose to the contralateral kidney and very minimal dose to the liver for patients with left-sided disease.

On-board kV-kV orthogonal pair images are used daily for patient alignment before treatment. During the course of treatment, daily cone beam CT (CBCT) is acquired to assess the soft tissue alignment and changes to patient anatomy (60). Any significant findings on the CBCT may prompt a re-scan on the CT simulator in order to have the dose calculated on the re-scanned CT. This is then evaluated by the physician and may trigger re-planning if undesired dosimetric parameters are observed. For centers who do not have on-board CBCT available, weekly re-scans with the CT simulator should be acquired and used for dosimetric assessment. On average, about half of the patients will require re-planning at least once during the treatment course.

\section{Conclusions}

MPM is a challenging disease to treat with radiation therapy, but improvements in surgical techniques and 


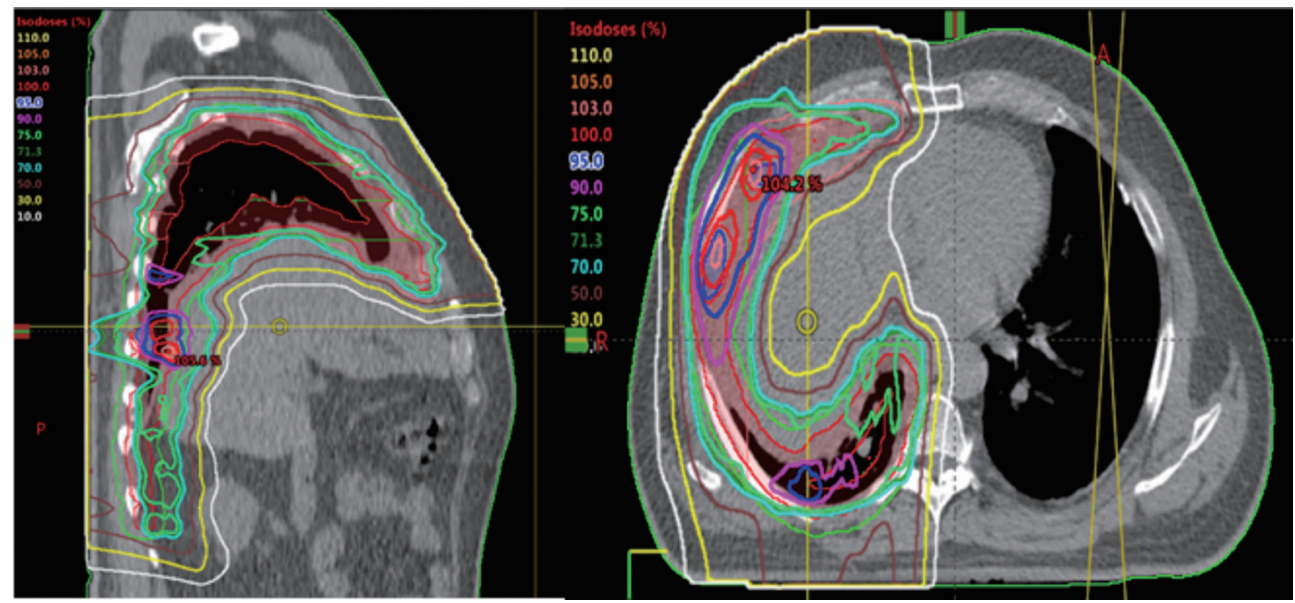

Figure 2 Sagittal and axial cross-sections of a patient with malignant pleural mesothelioma treated with a 2-field multi-field optimized (MFO) whole-pleural pencil-beam scanning proton therapy. The whole pleura was treated to 45 Gy in 25 fractions with simultaneous integrated boost to gross disease to $60 \mathrm{~Gy}$.

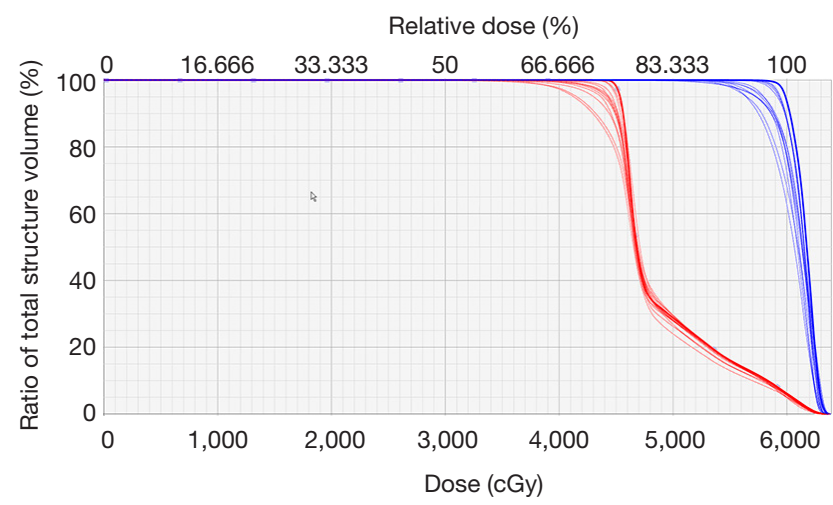

Figure 3 Dose-volume histogram with robustness evaluation showing dose to iCTV1 (red, 45 Gy in 25 fractions) and iCTV2 (blue, 60 Gy in 25 fractions). Nominal plan coverage is depicted with the solid lines and perturbed plans depicted in dotted lines. iCTV, internal clinical target volume.

systemic therapies as well as advancements in technology including the advent of PBS proton therapy have introduced the potential for a new paradigm in the treatment of MPM. Clinical trials will be needed to demonstrate the efficacy and safety of WP-IMPT alone and in combination with systemic therapies.

\section{Acknowledgements}

None.

\section{Footnote}

Conflicts of Interest: Dr. SN Badiyan and Dr. CB Simone 2nd have received speaker's honoraria from Varian Medical Systems. The other authors have no conflicts of interest to declare.

\section{References}

1. Ettinger DS, Akerley W, Borghaei H, et al. Malignant pleural mesothelioma. J Natl Compr Canc Netw 2012;10:26-41.

2. Howlander N, Noone AM, Krapcho M, et al. SEER Cancer Statistics Review, 1975-2013, National Cancer Institute. Available online: http://seer.cancer.gov/ csr/1975_2013

3. Carbone M, Ly BH, Dodson RF, et al. Malignant mesothelioma: facts, myths, and hypotheses. J Cell Physiol 2012;227:44-58.

4. Carbone M, Ferris LK, Baumann F, et al. BAP1 cancer syndrome: malignant mesothelioma, uveal and cutaneous melanoma, and MBAITs. J Transl Med 2012;10:179.

5. Chirieac LR, Barletta JA, Yeap BY, et al. Clinicopathologic characteristics of malignant mesotheliomas arising in patients with a history of radiation for Hodgkin and nonHodgkin lymphoma. J Clin Oncol 2013;31:4544-9.

6. De Bruin ML, Burgers JA, Baas P, et al. Malignant mesothelioma after radiation treatment for Hodgkin lymphoma. Blood 2009;113:3679-81. 
7. van Zandwijk N, Clarke C, Henderson D, et al. Guidelines for the diagnosis and treatment of malignant pleural mesothelioma. J Thorac Dis 2013;5:E254-307.

8. Beebe-Dimmer JL, Fryzek JP, Yee CL, et al. Mesothelioma in the United States: a Surveillance, Epidemiology, and End Results (SEER)-Medicare investigation of treatment patterns and overall survival. Clin Epidemiol 2016;8:743-50.

9. Scherpereel A, Astoul P, Baas P, et al. Guidelines of the European Respiratory Society and the European Society of Thoracic Surgeons for the management of malignant pleural mesothelioma. Eur Respir J 2010;35:479-95.

10. Tsao AS, Wistuba I, Roth JA, et al. Malignant pleural mesothelioma. J Clin Oncol 2009;27:2081-90.

11. Wang S, Ma K, Wang Q, et al. The revised staging system for malignant pleural mesothelioma based on surveillance, epidemiology, and end results database. Int J Surg 2017;48:92-8.

12. Nowak AK, Chansky K, Rice DC, et al. The IASLC Mesothelioma Staging Project: Proposals for Revisions of the T Descriptors in the Forthcoming Eighth Edition of the TNM Classification for Pleural Mesothelioma. J Thorac Oncol 2016;11:2089-99.

13. Rusch VW, Venkatraman E. The importance of surgical staging in the treatment of malignant pleural mesothelioma. J Thorac Cardiovasc Surg 1996;111:81525; discussion 825-6.

14. Sugarbaker DJ, Flores RM, Jaklitsch MT, et al. Resection margins, extrapleural nodal status, and cell type determine postoperative long-term survival in trimodality therapy of malignant pleural mesothelioma: results in 183 patients. J Thorac Cardiovasc Surg 1999;117:54-63; discussion 63-5.

15. Bille A, Platania M, Pelosi G, et al. Gingival metastasis as first sign of multiorgan dissemination of epithelioid malignant mesothelioma. J Thorac Oncol 2014;9:1226-9.

16. Ward RE, Ali SA, Kuhar M. Epithelioid malignant mesothelioma metastatic to the skin: A case report and review of the literature. J Cutan Pathol 2017;44:1057-63.

17. Miller AC, Miettinen M, Schrump DS, et al. Malignant mesothelioma and central nervous system metastases. Report of two cases, pooled analysis, and systematic review. Ann Am Thorac Soc 2014;11:1075-81.

18. van Meerbeeck JP, Scherpereel A, Surmont VF, et al. Malignant pleural mesothelioma: the standard of care and challenges for future management. Crit Rev Oncol Hematol 2011;78:92-111.

19. Chen J, Li Z, Xu N, et al. Efficacy of medical thoracoscopic talc pleurodesis in malignant pleural effusion caused by different types of tumors and different pathological classifications of lung cancer. Int J Clin Exp Med 2015;8:18945-53.

20. Wald O, Sugarbaker DJ. New Concepts in the Treatment of Malignant Pleural Mesothelioma. Annu Rev Med 2018;69:365-77.

21. Verma V, Ahern CA, Berlind CG, et al. National Cancer Database Report on Pneumonectomy Versus LungSparing Surgery for Malignant Pleural Mesothelioma. J Thorac Oncol 2017;12:1704-14.

22. Batirel HF. Extrapleural pneumonectomy (EPP) vs. pleurectomy decortication (P/D). Ann Transl Med 2017;5:232.

23. Simone CB 2nd, Cengel KA. Photodynamic therapy for lung cancer and malignant pleural mesothelioma. Semin Oncol 2014;41:820-30.

24. Friedberg JS, Simone CB 2nd, Culligan MJ, et al. Extended Pleurectomy-Decortication-Based Treatment for Advanced Stage Epithelial Mesothelioma Yielding a Median Survival of Nearly Three Years. Ann Thorac Surg 2017;103:912-9.

25. Flores RM, Pass HI, Seshan VE, et al. Extrapleural pneumonectomy versus pleurectomy/decortication in the surgical management of malignant pleural mesothelioma: results in 663 patients. J Thorac Cardiovasc Surg 2008;135:620-6, 626.e1-3.

26. Vogelzang NJ, Rusthoven JJ, Symanowski J, et al. Phase III study of pemetrexed in combination with cisplatin versus cisplatin alone in patients with malignant pleural mesothelioma. J Clin Oncol 2003;21:2636-44.

27. Zalcman G, Mazieres J, Margery J, et al. Bevacizumab for newly diagnosed pleural mesothelioma in the Mesothelioma Avastin Cisplatin Pemetrexed Study (MAPS): a randomised, controlled, open-label, phase 3 trial. Lancet 2016;387:1405-14.

28. Alley EW, Lopez J, Santoro A, et al. Clinical safety and activity of pembrolizumab in patients with malignant pleural mesothelioma (KEYNOTE-028): preliminary results from a non-randomised, open-label, phase $1 \mathrm{~b}$ trial. Lancet Oncol 2017;18:623-30.

29. Carmichael J, Degraff WG, Gamson J, et al. Radiation sensitivity of human lung cancer cell lines. Eur J Cancer Clin Oncol 1989;25:527-34.

30. Hakkinen AM, Laasonen A, Linnainmaa K, et al. Radiosensitivity of mesothelioma cell lines. Acta Oncol 1996;35:451-6.

31. Gordon W Jr, Antman KH, Greenberger JS, et al. Radiation therapy in the management of patients with mesothelioma. Int J Radiat Oncol Biol Phys 1982;8:19-25. 
32. de Graaf-Strukowska L, van der Zee J, van Putten W, et al. Factors influencing the outcome of radiotherapy in malignant mesothelioma of the pleura--a single-institution experience with 189 patients. Int J Radiat Oncol Biol Phys 1999;43:511-6.

33. Boutin C, Rey F, Viallat JR. Prevention of malignant seeding after invasive diagnostic procedures in patients with pleural mesothelioma. A randomized trial of local radiotherapy. Chest 1995;108:754-8.

34. O'Rourke N, Garcia JC, Paul J, et al. A randomised controlled trial of intervention site radiotherapy in malignant pleural mesothelioma. Radiother Oncol 2007;84:18-22.

35. Bydder S, Phillips M, Joseph DJ, et al. A randomised trial of single-dose radiotherapy to prevent procedure tract metastasis by malignant mesothelioma. Br J Cancer 2004;91:9-10.

36. de Perrot M, Feld R, Leighl NB, et al. Accelerated hemithoracic radiation followed by extrapleural pneumonectomy for malignant pleural mesothelioma. J Thorac Cardiovasc Surg 2016;151:468-73.

37. Yajnik S, Rosenzweig KE, Mychalczak B, et al. Hemithoracic radiation after extrapleural pneumonectomy for malignant pleural mesothelioma. Int J Radiat Oncol Biol Phys 2003;56:1319-26.

38. Krayenbuehl J, Oertel S, Davis JB, et al. Combined photon and electron three-dimensional conformal versus intensity-modulated radiotherapy with integrated boost for adjuvant treatment of malignant pleural mesothelioma after pleuropneumonectomy. Int J Radiat Oncol Biol Phys 2007;69:1593-9.

39. Miles EF, Larrier NA, Kelsey CR, et al. Intensitymodulated radiotherapy for resected mesothelioma: the Duke experience. Int J Radiat Oncol Biol Phys 2008;71:1143-50.

40. Patel PR, Yoo S, Broadwater G, et al. Effect of increasing experience on dosimetric and clinical outcomes in the management of malignant pleural mesothelioma with intensity-modulated radiation therapy. Int J Radiat Oncol Biol Phys 2012;83:362-8.

41. Giraud P, Sylvestre A, Zefkili S, et al. Helical tomotherapy for resected malignant pleural mesothelioma: dosimetric evaluation and toxicity. Radiother Oncol 2011;101:303-6.

42. de Perrot M, Feld R, Cho BC, et al. Trimodality therapy with induction chemotherapy followed by extrapleural pneumonectomy and adjuvant high-dose hemithoracic radiation for malignant pleural mesothelioma. J Clin Oncol 2009;27:1413-8.
43. Gomez DR, Hong DS, Allen PK, et al. Patterns of failure, toxicity, and survival after extrapleural pneumonectomy and hemithoracic intensity-modulated radiation therapy for malignant pleural mesothelioma. J Thorac Oncol 2013;8:238-45.

44. van Sandick JW, Kappers I, Baas P, et al. Surgical treatment in the management of malignant pleural mesothelioma: a single institution's experience. Ann Surg Oncol 2008; 15:1757-64.

45. Tonoli S, Vitali P, Scotti V, et al. Adjuvant radiotherapy after extrapleural pneumonectomy for mesothelioma. Prospective analysis of a multi-institutional series. Radiother Oncol 2011;101:311-5.

46. Allen AM, Czerminska M, Janne PA, et al. Fatal pneumonitis associated with intensity-modulated radiation therapy for mesothelioma. Int J Radiat Oncol Biol Phys 2006;65:640-5.

47. Kristensen CA, Nottrup TJ, Berthelsen AK, et al. Pulmonary toxicity following IMRT after extrapleural pneumonectomy for malignant pleural mesothelioma. Radiother Oncol 2009;92:96-9.

48. Rice DC, Smythe WR, Liao Z, et al. Dose-dependent pulmonary toxicity after postoperative intensity-modulated radiotherapy for malignant pleural mesothelioma. Int J Radiat Oncol Biol Phys 2007;69:350-7.

49. Stahel RA, Riesterer O, Xyrafas A, et al. Neoadjuvant chemotherapy and extrapleural pneumonectomy of malignant pleural mesothelioma with or without hemithoracic radiotherapy (SAKK 17/04): a randomised, international, multicentre phase 2 trial. Lancet Oncol 2015;16:1651-8.

50. Kutcher GJ, Kestler C, Greenblatt D, et al. Technique for external beam treatment for mesothelioma. Int J Radiat Oncol Biol Phys 1987;13:1747-52.

51. Gupta V, Mychalczak B, Krug L, et al. Hemithoracic radiation therapy after pleurectomy/decortication for malignant pleural mesothelioma. Int J Radiat Oncol Biol Phys 2005;63:1045-52.

52. Rosenzweig KE, Zauderer MG, Laser B, et al. Pleural intensity-modulated radiotherapy for malignant pleural mesothelioma. Int J Radiat Oncol Biol Phys 2012;83:1278-83.

53. Rimner A, Spratt DE, Zauderer MG, et al. Failure patterns after hemithoracic pleural intensity modulated radiation therapy for malignant pleural mesothelioma. Int J Radiat Oncol Biol Phys 2014;90:394-401.

54. Rimner A, Zauderer MG, Gomez DR, et al. Phase II Study of Hemithoracic Intensity-Modulated Pleural 
Radiation Therapy (IMPRINT) As Part of Lung-Sparing Multimodality Therapy in Patients With Malignant Pleural Mesothelioma. J Clin Oncol 2016;34:2761-8.

55. Minatel E, Trovo M, Polesel J, et al. Tomotherapy after pleurectomy/decortication or biopsy for malignant pleural mesothelioma allows the delivery of high dose of radiation in patients with intact lung. J Thorac Oncol 2012;7:1862-6.

56. Kishan AU, Cameron RB, Wang PC, et al. Tomotherapy improves local control and changes failure patterns in locally advanced malignant pleural mesothelioma. Pract Radiat Oncol 2015;5:366-73.

57. Harrabi SB, Koerber SA, Adeberg S, et al. Malignant pleural mesothelioma - Pleural cavity irradiation after decortication with helical tomotherapy. Rep Pract Oncol Radiother 2017;22:402-7.

58. Lomax AJ. Physics of Treatment Planning Using Scanned Beams. Proton Therapy Physics. Boca Raton, FL: CRC, 2011.

59. Kang M, Huang S, Solberg TD, et al. A study of the beamspecific interplay effect in proton pencil beam scanning delivery in lung cancer. Acta Oncol 2017;56:531-40.

60. Veiga C, Janssens G, Teng CL, et al. First Clinical Investigation of Cone Beam Computed Tomography and Deformable Registration for Adaptive Proton Therapy for Lung Cancer. Int J Radiat Oncol Biol Phys 2016;95:549-59.

61. Krayenbuehl J, Hartmann M, Lomax AJ, et al. Proton therapy for malignant pleural mesothelioma after extrapleural pleuropneumonectomy. Int J Radiat Oncol Biol Phys 2010;78:628-34.

62. Lorentini S, Amichetti M, Spiazzi L, et al. Adjuvant intensity-modulated proton therapy in malignant pleural mesothelioma. A comparison with intensity-modulated radiotherapy and a spot size variation assessment. Strahlenther Onkol 2012;188:216-25.

63. Verma V, Simone CB 2nd, Mishra MV. Quality of Life and Patient-Reported Outcomes Following Proton Radiation Therapy: A Systematic Review. J Natl Cancer Inst 2018;110. doi: 10.1093/jnci/djx208.

64. Chang JY, Zhang X, Knopf A, et al. Consensus Guidelines for Implementing Pencil-Beam Scanning Proton Therapy

Cite this article as: Badiyan SN, Molitoris JK, Zhu M, Glass E, Diwanji T, Simone CB 2nd. Proton beam therapy for malignant pleural mesothelioma. Transl Lung Cancer Res 2018;7(2):189198. doi: $10.21037 /$ tlcr.2018.04.07 for Thoracic Malignancies on Behalf of the PTCOG Thoracic and Lymphoma Subcommittee. Int J Radiat Oncol Biol Phys 2017;99:41-50.

65. Higgins KA, O'Connell K2, Liu Y, et al. National Cancer Database Analysis of Proton Versus Photon Radiation Therapy in Non-Small Cell Lung Cancer. Int J Radiat Oncol Biol Phys 2017;97:128-37.

66. Chang JY, Verma V, Li M, et al. Proton Beam Radiotherapy and Concurrent Chemotherapy for Unresectable Stage III Non-Small Cell Lung Cancer: Final Results of a Phase 2 Study. JAMA Oncol 2017;3:e172032.

67. Remick JS, Schonewolf C, Gabriel P, et al. First Clinical Report of Proton Beam Therapy for Postoperative Radiotherapy for Non-Small-Cell Lung Cancer. Clin Lung Cancer 2017;18:364-71.

68. Rwigema JM, Verma V, Lin L, et al. Prospective study of proton-beam radiation therapy for limited-stage small cell lung cancer. Cancer 2017;123:4244-51.

69. Vogel J, Berman AT, Lin L, et al. Prospective study of proton beam radiation therapy for adjuvant and definitive treatment of thymoma and thymic carcinoma: Early response and toxicity assessment. Radiother Oncol 2016;118:504-9.

70. Chao HH, Berman AT, Simone CB 2nd, et al. MultiInstitutional Prospective Study of Reirradiation with Proton Beam Radiotherapy for Locoregionally Recurrent Non-Small Cell Lung Cancer. J Thorac Oncol 2017;12:281-92.

71. Pan HY, Jiang S, Sutton J, et al. Early experience with intensity modulated proton therapy for lungintact mesothelioma: A case series. Pract Radiat Oncol 2015;5:e345-53.

72. Li YR, Alley EW, Friedberg JS, et al. editors. Prospective Assessment of Proton Therapy for Malignant Pleural Mesothelioma. International Association for the Study of Lung Cancer 16th World Conference on Lung Cancer, 2015.

73. Lee H, Zeng J, Bowen SR, et al. Proton Therapy for Malignant Pleural Mesothelioma: A Three Case Series Describing the Clinical and Dosimetric Advantages of Proton-Based Therapy. Cureus 2017;9:e1705. 